\title{
Asteroseismological constraints on the coolest GW Virginis variable star (PG 1159-type) PG 0122+200
}

\author{
A. H. Córsico ${ }^{1,2, \star}$, M. M. Miller Bertolami ${ }^{1,2, \star \star}$, L. G. Althaus ${ }^{1,2, \star}$, G. Vauclair $^{3}$, and K. Werner ${ }^{4}$ \\ ${ }^{1}$ Facultad de Ciencias Astronómicas y Geofísicas, Universidad Nacional de La Plata, Paseo del Bosque S/N, (1900) La Plata, \\ Argentina \\ e-mail: [acorsico;althaus;mmiller]@fcaglp.unlp.edu.ar \\ 2 Instituto de Astrofísica La Plata, IALP, CONICET-UNLP, Argentina \\ 3 Université Paul Sabatier, Observatoire Midi-Pyrénées, CNRS/UMR5572, 14 Av. Belin, 31400 Toulouse, France \\ e-mail: gerardv@ast.obs-mip.fr \\ 4 Institut für Astronomie und Astrophysik, Universität Tübingen, Sand 1, 72076 Tübingen, Germany \\ e-mail: werner@astro.uni-tuebingen.de
}

Received 22 June 2007 / Accepted 29 August 2007

\begin{abstract}
Aims. We present an asteroseismological study of PG $0122+200$, the coolest known pulsating PG 1159 (GW Vir) star. Our results are based on an augmented set of the full PG 1159 evolutionary models recently presented by Miller Bertolami \& Althaus (2006).

Methods. We perform extensive computations of adiabatic $g$-mode pulsation periods on PG 1159 evolutionary models with stellar masses ranging from 0.530 to $0.741 M_{\odot}$. These models take into account the complete evolution of progenitor stars, through the thermally pulsing asymptotic giant branch phase and born-again episode. We constrain the stellar mass of PG $0122+200$ by comparing the observed period spacing with the asymptotic period spacing and with the average of the computed period spacings. We also employ the individual observed periods to find a representative seismological model for PG $0122+200$.

Results. We derive a stellar mass of $0.626 M_{\odot}$ from a comparison between the observed period spacing and the computed asymptotic period spacing, and a stellar mass of $0.567 M_{\odot}$ by comparing the observed period spacing with the average of the computed period spacing. We also find, on the basis of a period-fit procedure, an asteroseismological model representative of PG $0122+200$ that is able to reproduce the observed period pattern with an average of the period differences of $\overline{\delta \Pi_{i}}=0.88 \mathrm{~s}$ and a root-mean-square residual of $\sigma_{\delta \Pi_{i}}=1.27 \mathrm{~s}$. The model has an effective temperature $T_{\text {eff }}=81500 \mathrm{~K}$, a stellar mass $M_{*}=0.556 M_{\odot}$, a surface gravity $\log g=7.65$, a stellar luminosity and radius of $\log \left(L_{*} / L_{\odot}\right)=1.14$ and $\log \left(R_{*} / R_{\odot}\right)=-1.73$, respectively, and a He-rich envelope thickness of $M_{\text {env }}=1.9 \times 10^{-2} M_{\odot}$. We derive a seismic distance $d \sim 614 \mathrm{pc}$ and a parallax $\pi \sim 1.6$ mas. The results of the period-fit analysis carried out in this work suggest that the asteroseismological mass of PG $0122+200$ could be $\sim 6-20 \%$ lower than hitherto thought, and in closer agreement (to within $\sim 5 \%$ ) with the spectroscopic mass. This result suggests that a reasonable consistency between the stellar mass values obtained from spectroscopy and asteroseismology can be expected when detailed PG 1159 evolutionary models are considered.
\end{abstract}

Key words. stars: evolution - stars: interiors - stars: oscillations - stars: variables: general - stars: white dwarfs

\section{Introduction}

PG $0122+200$ (BB Psc or WD 0122+200) is the coolest known pulsating PG 1159 star belonging to the GW Vir class of variables. GW Vir stars are very hot hydrogen-deficient postasymptotic giant branch (AGB) stars with surface layers rich in helium, carbon and oxygen (Werner \& Herwig 2006). They exhibit multiperiodic luminosity variations with periods in the range 5-50 min, attributable to nonradial pulsation $g$-modes. PG 1159 stars are thought to be the evolutionary link between Wolf-Rayet type central stars of planetary nebulae and most of the hydrogen-deficient white dwarfs. It is generally accepted that these stars have their origin in a born-again episode induced by a post-AGB helium thermal pulse (see Iben et al. 1983; Herwig et al. 1999; Lawlor \& MacDonald 2003; Althaus et al. 2005; Miller Bertolami et al. 2006, for recent references).

* Member of the Carrera del Investigador Científico y Tecnológico, CONICET, Argentina.

$\star \star$ Fellow of CONICET, Argentina
PG $0122+200$ is characterized by $T_{\text {eff }}=80000 \pm 4000 \mathrm{~K}$ and $\log g=7.5 \pm 0.5$ (Dreizler \& Heber 1998). At this effective temperature, PG $0122+200$ currently defines the locus of the low-luminosity red edge of the GW Vir instability strip. The photometric variations of this star were discovered by Bond \& Grauer (1987). Besides the intrinsic interest in probing its interior, pulsation studies of PG $0122+200$ offer a unique opportunity to study neutrino physics. Indeed, at the evolutionary stage characterizing PG $0122+200$, neutrino emission constitutes a main energy $\operatorname{sink}^{1}$ (O'Brien et al. 1998).

The determination of the stellar mass of PG $0122+200$ has been the subject of numerous investigations. The stellar mass of pulsating pre-white dwarfs can be constrained, in principle, from asteroseismology - the asteroseismological mass - either

\footnotetext{
1 At variance with the solar neutrino emission, which is a by-product of nuclear fusion, the neutrino flux of pre-white dwarf stars such as PG $0122+200$ is the result of different scattering processes, with the plasmaneutrino, Bremsstrahlung neutrino and photoneutrino emission being the most relevant ones (see O'Brien \& Kawaler 2000).
} 
through the observed period spacing (see, for instance, Kawaler \& Bradley 1994; Córsico \& Althaus 2006) or by means of the individual observed periods (see, e.g., Kawaler \& Bradley 1994; Córsico \& Althaus 2006; Córsico et al. 2007). The early study of O'Brien et al. (1996) predicts a stellar mass of about 0.66-0.72 $M_{\odot}$ for PG $0122+200$, corresponding to an observed mean period spacing of $21.2 \mathrm{~s}$. Vauclair et al. (1995), on the other hand, suggest an even higher stellar mass, based on a observed mean period spacing of PG $0122+200$ of $\sim 16$ s. Later, O'Brien et al. (1998) find strong evidence for a $\ell=1$ mean period spacing of $21 \mathrm{~s}$, although a value of $\sim 16 \mathrm{~s}$ cannot be conclusively ruled out. These values of the period spacing imply a stellar mass of $\sim 0.69 M_{\odot}$ and $\sim 1.0 M_{\odot}$, respectively, based on the PG 1159 models available at the time. By means of a period-fit procedure based on PG 1159 evolutionary models with several masses derived from the full sequence of $0.589 M_{\odot}$ of Althaus et al. (2005), Córsico \& Althaus (2006) obtain a stellar mass of $M_{*}=0.64 M_{\odot}$ for PG 0122+200. Recently, Fu et al. (2007) (hereinafter FUEA07) have presented new multisite photometric observations of PG $0122+200$ obtained in 2001 and 2002. By collecting the new data together with previous observations, these authors have succeeded in detecting a total of 23 frequencies corresponding to modes with $\ell=1$, and derived unambiguously a mean period spacing of $22.9 \mathrm{~s}$. On the basis of the models of Kawaler \& Bradley (1994), these authors inferred a stellar mass of $0.59 \pm 0.02 M_{\odot}$.

The stellar mass of PG 1159 stars can also be estimated through the comparison of the spectroscopic values of $T_{\text {eff }}$ and $g$ with evolutionary tracks - the spectroscopic mass. On the basis of the evolutionary tracks of O'Brien \& Kawaler (2000), Dreizler \& Heber (1998) derived a stellar mass of $0.53 \pm 0.1 M_{\odot}$ for PG 0122+200. On the other hand, Werner \& Herwig (2006) determined $M_{*}=0.58 M_{\odot}$ from a comparison with the H-rich evolutionary models of Schönberner (1983). The most recent determination is that of Miller Bertolami \& Althaus (2006), who derived a stellar mass of $0.53 M_{\odot}$ on the basis of PG 1159 evolutionary models that take fully into account the evolutionary history and the surface composition of the progenitor stars.

The discrepancy between the asteroseismological mass derived by FUEA07 $\left(0.57 \leq M_{*} / M_{\odot} \leq 0.61\right)$ and the most recent spectroscopic determination $\left(0.53 M_{\odot}\right)$ has prompted us to undertake the present asteroseismological investigation of PG $0122+200$, taking full advantage of the new generation of PG 1159 evolutionary models recently developed by Miller Bertolami \& Althaus (2006). These authors have followed in detail all of the evolutionary phases prior to the formation of PG 1159 stars with different stellar masses, particularly the born-again stage. In addition to the issue of the stellar mass, the employment of such detailed PG 1159 models allows us to address the question of the He-rich envelope mass $\left(q_{y} \equiv M_{\mathrm{env}} / M_{\odot}\right)$ of PG $0122+200$, which FUEA07 constrain to be in the range $-6 \lesssim \log q_{y} \lesssim-5.3$. Finally, a precise knowledge of the mass of PG $0122+200$ is a crucial aspect for determining the role played by neutrinos in that star.

The paper is organized as follows. In the next section we briefly describe our PG 1159 evolutionary models. In Sect. 3 we derive the stellar mass of PG $0122+200$ by means of the observed period spacing. In Sect. 4 we derive structural parameters of this star by employing the individual observed periods; in this section we derive an asteroseismological model representative of PG 0122+200 (Sect. 4.1) and discuss its main structural and pulsational characteristics (Sect. 4.2), its helium envelope thickness (Sect. 4.3), its mode-trapping properties (Sect. 4.4) and the asteroseismological distance and parallax (Sect. 4.5). Finally, in

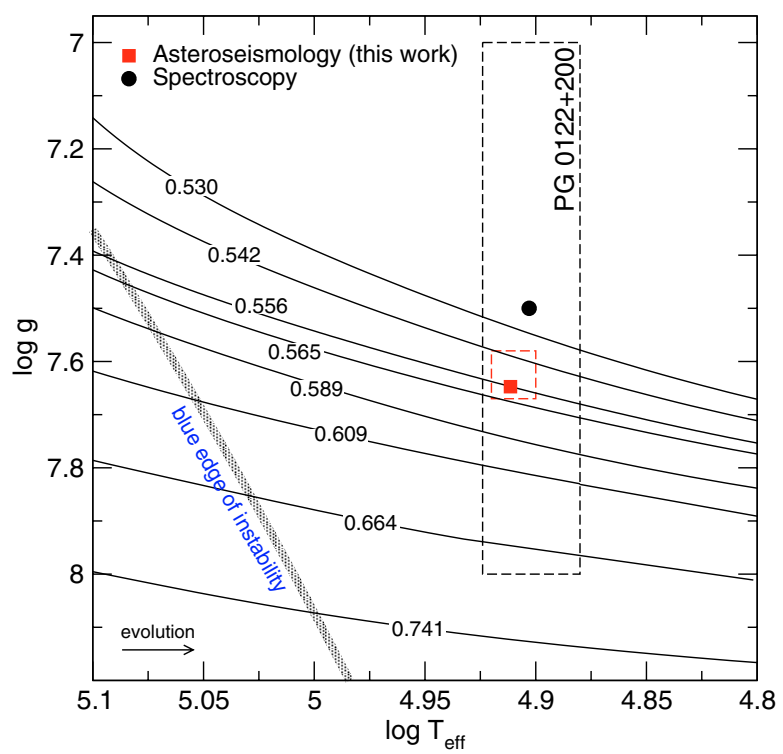

Fig. 1. Our PG 1159 full evolutionary tracks in the $\log T_{\text {eff }}-\log g$ plane, labelled with the corresponding stellar mass value in solar units. The black circle is the location of PG $0122+200$ according to spectroscopy $\left(T_{\text {eff }}=80 \pm 4 \mathrm{kK}\right.$ and $\left.\log g=7.5 \pm 0.5\right)$. Note the large error box (dashed), in particular for $\log g$. The square (red) is the location of the star as predicted by our asteroseismological analysis (see Sect. 4). The blue (hot) boundary of the theoretical dipole $(\ell=1)$ instability domain according to Córsico et al. (2006) - is also shown. (Color figure only available in the electronic version of the article.)

Sect. 5 we summarize our main results and make some concluding remarks.

\section{Evolutionary models and numerical tools}

The pulsation analysis presented in this work relies on a new generation of stellar models that take into account the complete evolution of PG 1159 progenitor stars. These models have been recently employed by our group for a pulsation stability analysis of the GW Vir stars, and for an asteroseismological study of the hot PG 1159 star RX J2117.1+3412 (Córsico et al. 2006, 2007, respectively). The stages for the formation and evolution of PG 1159 stars were computed with the LPCODE evolutionary code, which is described at length in Althaus et al. (2005). The neutrino production rates adopted in our computations are those of Itoh et al. (1989, 1992).

Specifically, the background of stellar models was extracted from the evolutionary calculations recently presented by Althaus et al. (2005), Miller Bertolami \& Althaus (2006), and Córsico et al. (2006), who computed the complete evolution of model star sequences with initial masses on the ZAMS in the range 1-3.75 $M_{\odot}$. We refer the reader to those works for details. It should be noted that all of the post-AGB evolutionary sequences have been followed through the very late thermal pulse (VLTP) and the resulting born-again episode that gives rise to the H-deficient, helium-, carbon- and oxygen-rich composition characteristic of PG 1159 stars. The masses of the resulting remnants are $0.530,0.542,0.556,0.565,0.589,0.609,0.664$, and $0.741 M_{\odot}$. The new sequence with $M=0.556 M_{\odot}$, coming from a progenitor star with $M_{*}=1.8 M_{\odot}$ at the ZAMS, has been computed specifically for the present asteroseismological study. The evolutionary tracks in the $\log T_{\text {eff }}-\log g$ plane for the PG 1159 regime are displayed in Fig. 1. 


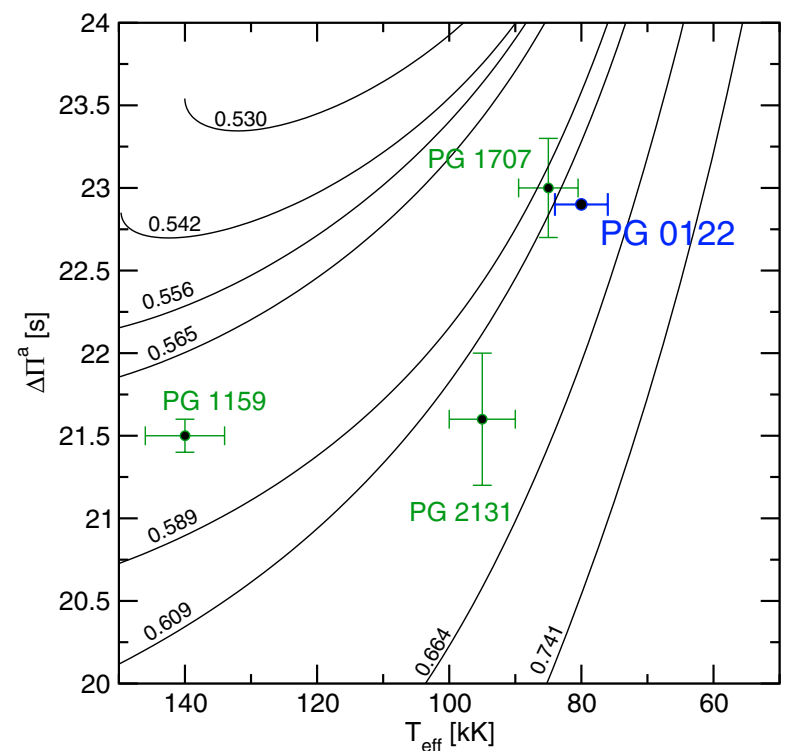

Fig. 2. The dipole $(\ell=1)$ asymptotic period spacing $\left(\Delta \Pi_{\ell}^{\mathrm{a}}\right)$ for different stellar masses in terms of the effective temperature. Numbers along each curve denote the stellar masses (in solar units). The plot also shows the location of PG $0122+200\left(T_{\text {eff }}=80 \pm 4 \mathrm{kK}\right.$ and $\left.\Delta \Pi^{\mathrm{O}}=22.9 \mathrm{~s}\right)$ and the remainder high-gravity, low-luminosity GW Vir stars (PG 1159-035, PG $2131+066$, and PG $1707+427$ ) with the period spacing and $T_{\text {eff }}$ data taken from Kawaler et al. (2004). The mass of PG $0122+200$ as derived by comparing $\Delta \Pi_{\ell}^{\mathrm{a}}$ with $\Delta \Pi^{\mathrm{O}}$ is $M_{*}=0.625_{-0.016}^{+0.019} M_{\odot}$.

It is worth mentioning that the use of these evolutionary tracks constitutes a major improvement with respect to previous asteroseismological studies. As mentioned, our PG 1159 evolutionary sequences are derived from the complete born-again evolution of progenitor stars and a careful treatment of the mixing and extramixing processes during the core helium burning; these are fundamental aspects when attempts are made at constructing stellar models appropriate for PG 1159 stars. In particular, these evolutionary calculations reproduce: (1) the spread in surface chemical composition observed in PG 1159 stars, (2) the short born-again times of V4334 Sgr (see Miller Bertolami et al. 2006; Miller Bertolami \& Althaus 2007a), (3) the location of the GW Vir instability strip in the $\log T_{\text {eff }}-\log g$ plane (Córsico et al. 2006), and (4) the expansion age of the planetary nebula of RX J2117.1+3412 (see the paper by Córsico et al. 2007, and its associated erratum). We believe that the employment of these new evolutionary computations render our pulsational inferences for PG 0122+200 more reliable.

We computed $\ell=1 g$-mode adiabatic pulsation periods and asymptotic period spacings with the same numerical code we employed in our previous works (see, e.g., Córsico \& Althaus 2006, for details). We analyzed about 3000 PG 1159 models covering a wide range of effective temperatures and luminosities $\left(5.4 \gtrsim \log \left(T_{\text {eff }}\right) \gtrsim 4.8\right.$ and $0 \lesssim \log \left(L_{*} / L_{\odot}\right) \lesssim 4.2$, respectivley $)$ and a range of stellar masses $\left(0.530 \leq M_{*} / M_{\odot} \leq 0.741\right)$.

\section{Mass determination from the observed period spacing}

Here, we constrain the stellar mass of PG $0122+200$ by comparing the asymptotic period spacing, $\Delta \Pi_{\ell}^{\mathrm{a}}$, and the average of the computed period spacings, $\overline{\Delta \Pi_{k}}$ ( $k$ being the radial order),

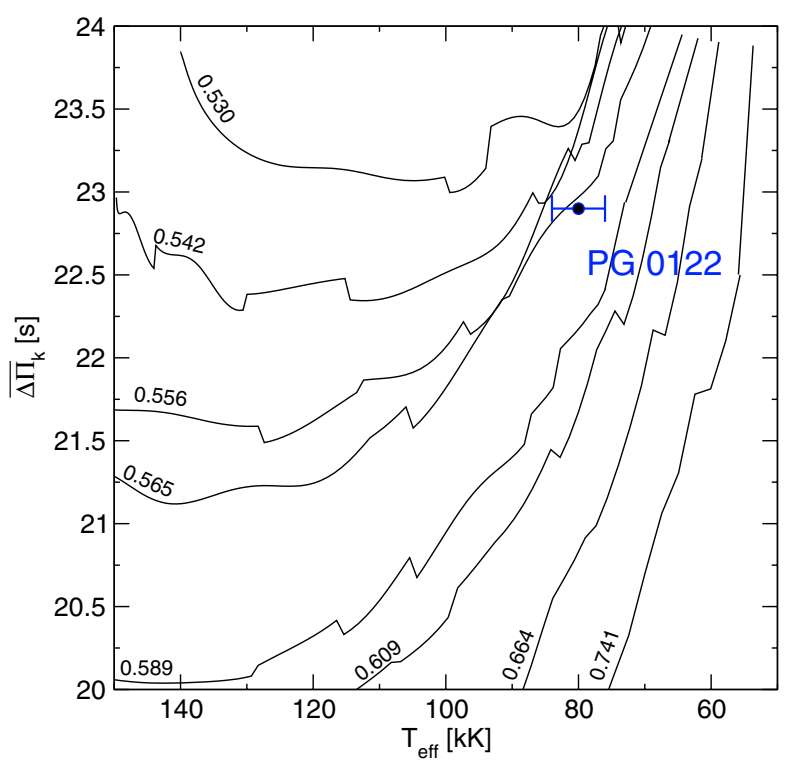

Fig. 3. Same as Fig. 2, but for the average of the computed period spacings $\left(\overline{\Delta \Pi_{k}}\right)$. The mass of PG $0122+200$ as derived by comparing $\overline{\Delta \Pi_{k}}$ with $\Delta \Pi^{\mathrm{O}}$ is $M_{*}=0.567_{-0.013}^{+0.007} M_{\odot}$.

with the observed period spacing, $\Delta \Pi^{\mathrm{O} 2}$. These methods take full advantage of the fact that the period spacing of PG 1159 pulsators depends primarily on the stellar mass, and weakly on the luminosity and the He-rich envelope mass fraction (Kawaler \& Bradley 1994; Córsico \& Althaus 2006). Note that in these approaches we make use of the spectroscopic constraint that the effective temperature of PG $0122+200$ is $80 \mathrm{kK}$ (Dreizler \& Heber 1998).

The asymptotic period spacing and the average of the computed period spacings for $\ell=1$ modes as a function of the effective temperature are displayed in Figs. 2 and 3, respectively, for different stellar masses. Also shown in these diagrams is the location of PG $0122+200$, with $\Delta \Pi^{\mathrm{O}}=22.9 \mathrm{~s}$ (FUEA07). Here, $\Delta \Pi_{\ell}^{\mathrm{a}}=\Pi_{0} / \sqrt{\ell(\ell+1)}$, where $\Pi_{0}=2 \pi^{2}\left[\int_{r_{1}}^{r_{2}}(N / r) \mathrm{d} r\right]^{-1}$, where $N$ is the Brunt-Väisälä frequency (Tassoul et al. 1990). The quantity $\overline{\Delta \Pi_{k}}$, on the other hand, is assessed by averaging the computed forward period spacings $\left(\Delta \Pi_{k}=\Pi_{k+1}-\Pi_{k}\right)$ in the range of the observed periods in PG $0122+200(330-620$ s; see Table 1).

From a comparison between $\Delta \Pi^{\mathrm{O}}$ and $\Delta \Pi_{\ell}^{\mathrm{a}}$ we obtain a stellar mass of $M_{*}=0.625_{-0.016}^{+0.019} M_{\odot}$. The quoted uncertainties in the value of $M_{*}$ come from the errors in the spectroscopic determination of the effective temperature. In the same way, we get $M_{*}=0.567_{-0.013}^{+0.007} M_{\odot}$ if we compare $\Delta \Pi^{\mathrm{O}}$ and $\overline{\Delta \Pi_{k}}$. A higher value of $M_{*}$ (about $10 \%$ larger) is derived from $\Delta \Pi_{\ell}^{\mathrm{a}}$, because the asymptotic period spacing is usually larger than the average of the computed period spacings (see Córsico \& Althaus 2006), in particular for the short periods like those exhibited by PG $0122+200$, i.e. those for which the full asymptotic regime of the modes $(k \gg 1)$ has not been attained ${ }^{3}$. It is important to note that the first method used to derive the stellar mass is somewhat less realistic than the second one, because the asymptotic

\footnotetext{
${ }^{2}$ Note that most asteroseismological studies rely on the asymptotic period spacing to infer the stellar mass of GW Vir pulsators.

3 At variance with this, for the longer periods exhibited by RX J2117.1+3412 (with $30 \leq k \leq 53$ ) the asymptotic conditions are more nearly reached and, as a result, the stellar mass derived from the asymptotic period spacing is very close to that derived from the average of the computed period spacings (see Córsico et al. 2007).
} 
predictions are, in principle, only valid for chemically homogeneous stellar models, while our PG 1159 models are chemically stratified.

Finally, we note that our inferred stellar mass values of $M_{*} \approx$ $0.57 M_{\odot}$ and, in particular, $M_{*} \approx 0.63 M_{\odot}$, are in conflict with the value $M_{*}=0.53 M_{\odot}$ as derived from spectroscopy coupled to evolutionary tracks (Dreizler \& Heber 1998; Miller Bertolami \& Althaus 2006).

\section{Constraints from the individual observed periods}

\subsection{The search for the best-fit model}

In this approach we seek the pulsation model that best matches the individual pulsation periods of PG $0122+200$. We assume that all of the observed periods correspond to $\ell=1$ modes (see FUEA07). The goodness of the match between the theoretical pulsation periods $\left(\Pi_{k}\right)$ and the observed individual periods $\left(\Pi_{i}^{\mathrm{O}}\right)$ is measured by means of a quality function defined as $\chi^{2}\left(M_{*}, T_{\text {eff }}\right)=\sum_{i=1}^{n} \min \left[\left(\Pi_{i}^{\mathrm{O}}-\Pi_{k}\right)^{2}\right] / n$, where $n(=9)$ is the number of observed periods (first column in Table 1). The PG 1159 model that shows the lowest value of $\chi^{2}$ will be adopted as the "best-fit model". This approach has also been used by Córsico $\&$ Althaus (2006) and Córsico et al. (2007).

We evaluate the function $\chi^{2}\left(M_{*}, T_{\text {eff }}\right)$ for stellar masses of $0.530,0.542,0.556,0.565,0.589,0.609,0.664$, and $0.741 M_{\odot}$. For the effective temperature we employed a much finer grid $\left(\Delta T_{\text {eff }}=10-30 \mathrm{~K}\right)$. The quantity $\left(\chi^{2}\right)^{-1}$, in terms of the effective temperature for different stellar masses, is shown in Fig. 4 together with the spectroscopic effective temperature of PG 0122+200. We find one strong maximum for the model with $M_{*}=0.556 M_{\odot}$ and $T_{\text {eff }} \approx 81.5 \mathrm{kK}$ (panel c). Such a pronounced maximum in the inverse of $\chi^{2}$ implies an excellent agreement between the theoretical and observed periods. Another maximum, albeit somewhat less pronounced, is encountered for the model with $M_{*}=0.542 M_{\odot}$ at $T_{\text {eff }} \approx 87.7 \mathrm{kK}$ and constitutes another acceptable asteroseismological solution, in particular because its stellar mass is closer to the spectroscopic mass of PG $0122+200$ $\left(0.53 M_{\odot}\right)$. However, because the agreement between observed and theoretical periods for this model is somewhat poorer than for the one with $M_{*}=0.556 M_{\odot}$, we choose to adopt this last model as the best-fit asteroseismological model. Note that our best-fit model has an effective temperature very close to that suggested by spectroscopy, well inside the error bar. A detailed comparison of the observed $m=0$ periods in PG $0122+200$ with the theoretical periods of the best-fit model is provided in Table 1 . The high quality of our period fit is quantitatively reflected by the average of the absolute period differences, $\overline{\delta \Pi_{i}}=\left(\sum_{i=1}^{n}\left|\delta \Pi_{i}\right|\right) / n$, where $\delta \Pi_{i}=\Pi_{i}^{\mathrm{O}}-\Pi_{k}$, and by the root-mean-square residual, $\sigma_{\delta \Pi_{i}}=\sqrt{\left(\sum\left|\delta \Pi_{i}\right|^{2}\right) / n}$. We obtain $\overline{\delta \Pi_{i}}=0.88 \mathrm{~s}$ and $\sigma_{\delta \Pi_{i}}=1.27 \mathrm{~s}$. The quality of our fit for PG $0122+200$ is much better than that achieved by Córsico et al. (2007) for RX J2117.1+3412 $\left(\overline{\delta \Pi_{i}}=1.08 \mathrm{~s}\right)$, and those obtained by Kawaler \& Bradley (1994) and Córsico \& Althaus (2006) $\overline{\left(\delta \Pi_{i}\right.}=1.19 \mathrm{~s}$ and $\overline{\delta \Pi_{i}}=1.79 \mathrm{~s}$, respectively) for PG 1159-035. Note that we are able to get a PG 1159 model that reproduces the period spectrum observed in PG $0122+200$ without artificially tuning the value of structural parameters such as the thickness of the outer envelope, the surface chemical abundances, or the shape of the core chemical profile which are, instead, kept fixed at the values predicted by the evolutionary computations.

Table 1 also shows the linear growth rates $\left(\eta_{k}\right)$ of the fitted pulsation modes (fifth column) for our best-fit model, computed
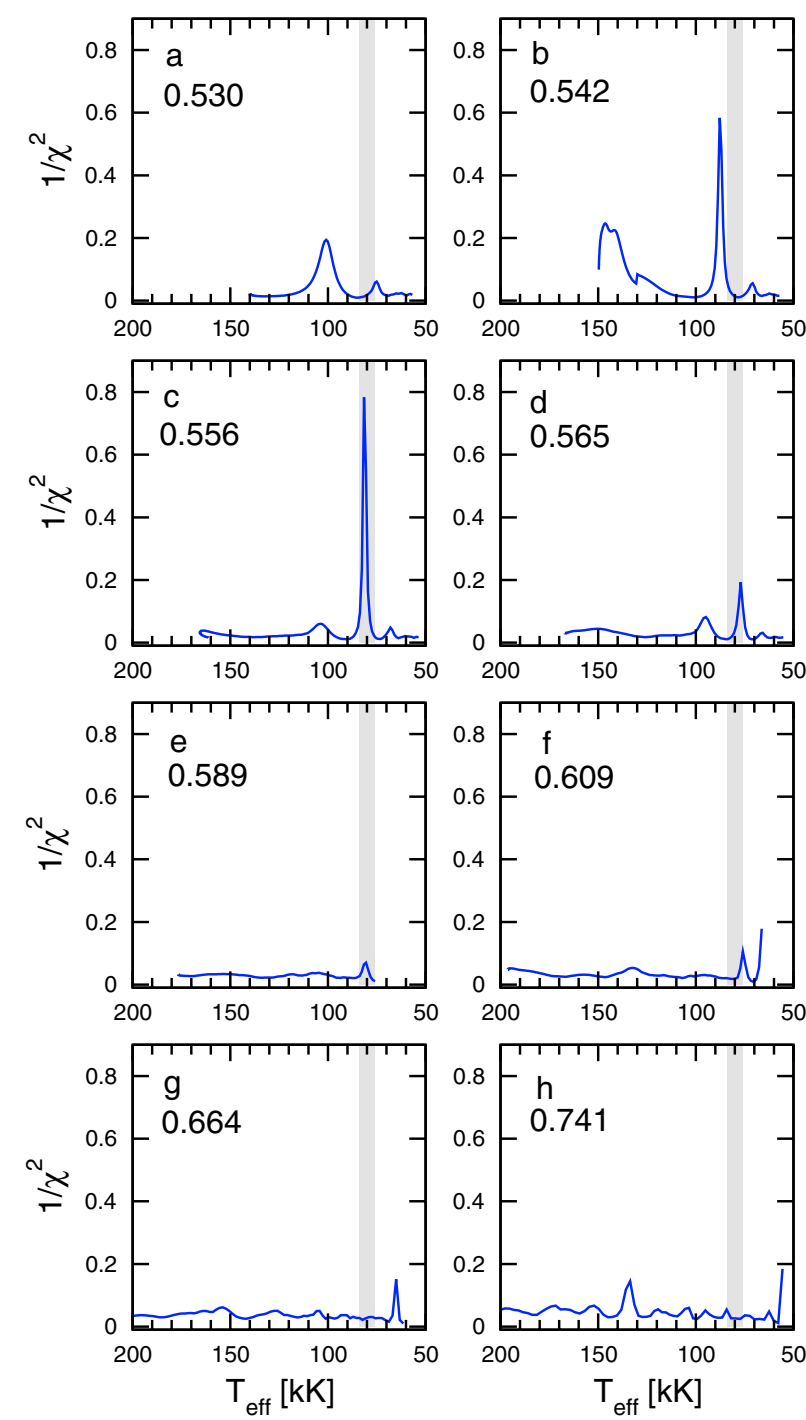

Fig. 4. The inverse of the quality function of the period fit in terms of the effective temperature for the PG 1159 sequences with different stellar masses indicated (in solar mass) in each panel. The grey vertical strip corresponds to the spectroscopic effective temperature of PG $0122+200$ and its uncertainties $\left(T_{\text {eff }}=80000 \pm 4000 \mathrm{~K}\right)$. Note the strong maximum in $\left(\chi^{2}\right)^{-1}$ for $M_{*}=0.556 M_{\odot}$ at $T_{\text {eff }} \approx 81500 \mathrm{~K}$. This corresponds to our "best-fit" model (see text for details). (Color figure only available in the electronic version of the article.)

with the nonadiabatic pulsation code described in Córsico et al. (2006). We found that all of the fitted modes have positive values of $\eta_{k}$, implying pulsational instability, although our stability analysis predicts a band of unstable mode-periods $\left(230 \lesssim \Pi_{k} \lesssim\right.$ $730 \mathrm{~s})$ somewhat wider than the interval of periods detected in PG 0122+200.

The last column in Table 1 shows the rate of period change of the fitted pulsation modes. Our calculations predict all of the pulsation periods to increase with time $\left(\dot{\Pi}_{k}>0\right)$, in accordance with the decrease of the Brunt-Väisälä frequency in the core of the model induced by cooling. Note that at the effective temperature of PG $0122+200$, cooling has the largest effect on $\dot{\Pi}_{k}$, while gravitational contraction, which should result in a decrease of periods with time, becomes negligible and no longer affects the pulsation periods, except for the case of modes trapped in the envelope (see Sect. 4.4). Until now, the only secure measurement of $\dot{\Pi}$ in pre-white dwarf stars is that of PG 1159-035, the 
Table 1. Observed $m=0$ periods $\left(\Pi_{i}^{\mathrm{O}}\right.$ ) for PG 0122+200 (taken from FUEA07), theoretical $\ell=1, m=0$ periods $\left(\Pi_{k}\right)$, period differences $\left(\delta \Pi_{i}=\Pi_{i}^{\mathrm{O}}-\Pi_{k}\right)$, radial orders $(k)$, linear growth rates $\left(\eta_{k}\right)$, and rates of period change $\left(\dot{\Pi}_{k}\right)$ for the best-fit model.

\begin{tabular}{cccccc}
\hline \hline $\begin{array}{c}\Pi_{i}^{\mathrm{O}} \\
{[\mathrm{s}]}\end{array}$ & $\begin{array}{c}\Pi_{k} \\
{[\mathrm{~s}]}\end{array}$ & $\begin{array}{c}\delta \Pi_{i} \\
{[\mathrm{~s}]}\end{array}$ & $k$ & $\begin{array}{c}\eta_{k} \\
{\left[10^{-6}\right]}\end{array}$ & $\begin{array}{c}\dot{\Pi}_{k} \\
{\left[10^{-12} \mathrm{~s} / \mathrm{s}\right]}\end{array}$ \\
\hline 336.68 & 334.12 & 2.56 & 12 & 1.12 & 1.22 \\
- & 354.85 & - & 13 & 1.74 & 1.70 \\
380.10 & 380.70 & -0.60 & 14 & 3.54 & 1.84 \\
400.99 & 400.35 & 0.64 & 15 & 6.13 & 1.59 \\
- & 425.25 & - & 16 & 8.53 & 2.24 \\
449.48 & 448.16 & 1.32 & 17 & 15.90 & 1.71 \\
468.69 & 469.32 & -0.63 & 18 & 16.30 & 2.46 \\
494.92 & 494.76 & 0.16 & 19 & 28.64 & 2.19 \\
517.96 & 516.65 & 1.31 & 20 & 36.50 & 2.10 \\
- & 539.65 & - & 21 & 32.24 & 3.11 \\
564.28 & 563.98 & 0.30 & 22 & 61.06 & 2.00 \\
- & 585.80 & - & 23 & 48.17 & 3.04 \\
611.15 & 610.73 & 0.42 & 24 & 55.99 & 3.26 \\
\hline
\end{tabular}

Table 2. The main characteristics of PG $0122+200$. The second column corresponds to spectroscopic results, whereas the third and fourth columns present results from the pulsation study of FUEA07 and from the asteroseismological model of this work, respectively.

\begin{tabular}{l|ccc}
\hline \hline Quantity & Spectroscopy & FUEA07 & $\begin{array}{c}\text { Asteroseismology } \\
\text { (This work) }\end{array}$ \\
\hline$T_{\text {eff }}[\mathrm{kK}]$ & $80 \pm 4^{(\mathrm{a})}$ & - & $81.54_{-1.4}^{+0.8}$ \\
$M_{*}\left[M_{\odot}\right]$ & $0.53 \pm 0.1^{(\mathrm{b})}$ & $0.59 \pm 0.02$ & $0.556_{-0.014}^{+0.009}$ \\
$\log g\left[\mathrm{~cm} / \mathrm{s}^{2}\right]$ & $7.5 \pm 0.5^{\text {(a) }}$ & - & $7.65_{-0.07}^{+0.02}$ \\
$\log \left(L_{*} / L_{\odot}\right)$ & $1.2_{-0.3}^{+0.2}(*)$ & $1.3 \pm 0.5$ & $1.14_{-0.04}^{+0.02}$ \\
$\log \left(R_{*} / R_{\odot}\right)$ & $-1.68_{-0.15}^{+0.10^{(*)}}$ & $-1.65 \pm 0.25$ & $-1.73_{-0.01}^{+0.025}$ \\
$M_{\text {env }}\left[M_{\odot}\right]$ & - & $(6-30) \times 10^{-7}$ & $0.019 \pm 0.006$ \\
$\mathrm{C} / \mathrm{He}, \mathrm{O} / \mathrm{He}^{(*)}$ & $0.9,0.4^{(\mathrm{a})}$ & - & $1.14,0.71$ \\
$\mathrm{BC}[\mathrm{mag}]$ & $-5.81_{-0.21}^{+0.23}$ & - & $-5.89_{-0.04}^{+0.08}$ \\
$M_{\mathrm{V}}[\mathrm{mag}]$ & $7.55_{-0.51}^{+0.74}$ & - & $7.79_{-0.10}^{+0.03}$ \\
$M_{\mathrm{bol}}[\mathrm{mag}]$ & 1.74 & - & $1.9_{-0.14}^{+0.11}$ \\
$A_{\mathrm{V}}[\mathrm{mag}]$ & 0.19 & - & 0.071 \\
$d[\mathrm{pc}]$ & 682 & $700_{-400}^{+1000}$ & $614_{-32}^{+58}$ \\
$\pi[\mathrm{mas}]$ & 1.47 & $1.43_{-0.84}^{+1.9}$ & $1.6 \pm 0.1$ \\
\hline
\end{tabular}

Note: $(*)$ Abundances by mass, $(* *)$ interpolated from the tracks by assuming spectroscopic $\left(T_{\text {eff }}, \log g\right)=(80 \mathrm{kK}, 7.5)$.

References: (a) Dreizler \& Heber (1998); (b) Miller Bertolami \& Althaus (2006).

prototype of the class, for which Costa et al. (1999) obtained a positive value of $\dot{\Pi}=(+1.307 \pm 0.003) \times 10^{-10} \mathrm{~s} / \mathrm{s}$ for the $516 \mathrm{~s}$ period. Note that our theoretical (positive) $\dot{\Pi}_{k}$ values for the bestfit model $\left(1.22-3.26 \times 10^{-12} \mathrm{~s} / \mathrm{s}\right)$ are two orders of magnitude lower. For the case of PG $0122+200$, a determination of any $\dot{\Pi}$ has not been assessed up to now, although work in this direction is in progress (see Fu et al. 2002).

\subsection{Characteristics of the best-fit model}

The main features of our best-fit model are summarized in Table 2, where we also include the parameters of PG $0122+200$ from other published studies ${ }^{4}$. Note that the effective temperature of our best-fit model is virtually the same as the

${ }^{4}$ Errors in $T_{\text {eff }}$ and $\log \left(L_{*} / L_{\odot}\right)$ are estimated from the width of the maximum in the function $\chi^{2}$ vs. $T_{\text {eff }}$ and $\log \left(L_{*} / L_{\odot}\right)$, respectively; the spectroscopic value. Thus, the location of the star in the $\log T_{\text {eff }}-\log g$ plane is vertically shifted to higher gravities according to our predictions (see Fig. 1).

Our best-fit model has a stellar mass of $M_{*}=0.556 M_{\odot}$, somewhat smaller than the value derived from the average of the computed period spacing, $M_{*} \sim 0.57 M_{\odot}$, and substantially lower than that inferred from the asymptotic period spacing, $M_{*} \sim 0.63 M_{\odot}$ (see Sect. 3). On the other hand, FUEA07 have inferred a value of the stellar mass of PG $0122+200$ by using an interpolation formula to the period spacing derived by Kawaler \& Bradley (1994) on the basis of a large grid of artificial PG 1159 models in the luminosity range $1.6 \lesssim \log \left(L_{*} / L_{\odot}\right) \lesssim 3.0$. These authors obtain a high value of $0.59 \pm 0.02 M_{\odot}$, in line with the trend of early determinations (O’Brien et al. 1998) and also in good agreement with our values derived from the period spacing, but in clear conflict with the mass of our best-fit model.

On the other hand, the $M_{*}$ value of our best-fit model is higher than the spectroscopic mass of $0.53 M_{\odot}$ derived by Miller Bertolami \& Althaus (2006) (see also Dreizler \& Heber 1998) for PG $0122+200$. Note that a discrepancy between the asteroseismological and the spectroscopic values of $M_{*}$ is generally encountered among PG 1159 pulsators (see Córsico et al. 2006, 2007). Until now, the asteroseismological mass of PG $0122+200$ has been about $10-30 \%$ larger $\left(\Delta M_{*} \approx\right.$ $0.06-0.17 M_{\odot}$ ) than the spectroscopic mass. In light of the bestfit model derived in this paper, this discrepancy is notably reduced to less than $5 \%\left(\Delta M_{*} \approx 0.026 M_{\odot}\right)$.

FUEA07 infer the stellar luminosity of PG $0122+200$ by using the formula of Kawaler \& Bradley (1994) mentioned above. They obtain $\log \left(L_{*} / L_{\odot}\right)=1.3 \pm 0.5$, larger than the luminosity of our best-fit model, $\log \left(L_{*} / L_{\odot}\right)=1.14_{-0.04}^{+0.02}$, and with significantly less accuracy. The large uncertainty in the luminosity quoted by FUEA07 is due to the large uncertainty in the spectroscopically determined $\log g$, a quantity used by these authors to derive the luminosity.

\subsection{Helium-rich envelope thickness}

An important parameter to be discussed separately is the thickness of the outer envelope $\left(M_{\text {env }}\right)$ of PG $0122+200$. We define $M_{\text {env }}$ as the mass above the chemical discontinuity between the He-rich envelope and the $\mathrm{C} / \mathrm{O}$ core. Our best-fit model has $M_{\text {env }}=0.019 M_{\odot}$. On the other hand, FUEA07 suggest a value of $M_{\text {env }}$ in the range $(6-30) \times 10^{-7} M_{\odot}$, about 5 orders of magnitude smaller. In this section, we try to answer the question: could a strikingly low value of $M_{\text {env }}$ like that suggested by FUEA07 be explained by mass loss during the PG 1159 phase? To this end, we performed additional PG 1159 evolutionary calculations to explore the amount of stellar mass that could be eroded by winds. Specifically, we have performed new evolutionary simulations for the sequence of the best-fit model $\left(M_{*}=0.556 M_{\odot}\right)$ starting from the second departure (post-VLTP) of the AGB until the PG 1159 stage is reached, with different mass loss rate prescriptions. Specifically, we have adopted two different mass loss rates $\left(\dot{M}_{1}, \dot{M}_{2}\right)$ appropriate for radiatively driven winds, namely, the one given by Blöcker (1995), which is based on Pauldrach et al. (1988), results

$\dot{M}_{1}=1.29 \times 10^{-15}\left(\frac{L_{*}}{L_{\odot}}\right)^{1.86}\left[M_{\odot} / \mathrm{yr}\right]$

error in the stellar mass comes from the grid resolution in $M_{*}$. Errors in the remainder quantities are derived from these values. 
and the one adopted by Lawlor \& MacDonald (2006), which is based on a modified version of the treatment of Abbott (1982),

$\dot{M}_{2}=1.2 \times 10^{-15}\left(\frac{L_{*}}{L_{\odot}}\right)^{2}\left(\frac{M_{\mathrm{eff}}}{M_{\odot}}\right)^{-1}\left(\frac{Z}{Z_{\odot}}\right)^{1 / 2}\left[M_{\odot} / \mathrm{yr}\right]$.

In the last expression $M_{\mathrm{eff}}=(1-\Gamma) M_{*}$ with $\Gamma$ defined as in Castor et al. (1975). The metallicity was set to $Z=Z_{\odot}$, because at high metallicities iron lines are expected to be dominant for radiative driven winds (Vink et al. 2001) and iron abundance is expected to remain unchanged during the whole evolution ${ }^{5} . \dot{M}_{1}$ is approximately one order of magnitude lower than $\dot{M}_{2}$ in the present simulations. The total amount of mass lost by these sequences when they reach the location of PG $0122+200$ is $7 \times 10^{-5} M_{\odot}$ for $\dot{M}_{1}$ and $4.4 \times 10^{-4} M_{\odot}$ for $\dot{M}_{2}$, which are both negligible as compared with the mass of the envelope of the best-fit model. For completeness we have considered a more extreme case by adopting a mass loss rate of $\dot{M}_{3}=10 \dot{M}_{2}$. In this case the mass loss rate at the WR-CSPN stage $\left(L_{*} \sim 10000 L_{\odot}\right.$ and $\left.T_{\text {eff }}<100000 \mathrm{~K}\right)$ is of the order of several $10^{-6} M_{\odot} / \mathrm{yr}$, and the rate at the evolutionary "knee" in the HR diagram during the PG 1159 stage is about $10^{-7} M_{\odot} / \mathrm{yr}$. These values are consistent with the largest rates observed at both the PG 1159 and WR-CSPN stages (Koesterke et al. 1998; Koesterke 2001) and, consequently, are probably an overestimation of the effect in view of the low mass of our best-fit model. Even in this case, the mass eroded by winds amounts to only $3.4 \times 10^{-3} M_{\odot}$ which is about one order of magnitude lower than the initial mass of the envelope ${ }^{6}$. Thus, it seems that envelopes as thin as those proposed by FUEA07 could be ruled out in the context of single star stellar evolution. More importantly, the reduction in the mass of the He-rich envelope from a canonical value of $\sim 10^{-2} M_{\odot}$ to a value of $\sim 10^{-7} M_{\odot}$ would require extreme fine-tuning (of five orders of magnitude) in the massloss rate to avoid the complete removal of the whole envelope. In the absence of a mechanism that justifies this fine-tuning, such extremely thin envelopes should be taken with some caution.

\subsection{Mode trapping}

In this section we shall try to disentangle the possible modetrapping signatures that could be hidden in the observed period spectrum of PG $0122+200$. Following FUEA07, we consider the residuals $\left(R_{\Pi}\right)$ of the period distribution relative to the mean period spacing ${ }^{7}$. For the case of PG $0122+200$, a linear leastsquare fit to the observed periods gives a mean period spacing of $22.97 \mathrm{~s}$, while for the best-fit model we obtain a mean theoretical period spacing of $23.05 \mathrm{~s}$. In panel a of Fig. 5 we plot the $R_{\Pi}$-distribution for the observed periods (red) and for the case of the calculated periods (black) of the best fit model. The calculated distribution is in very good agreement with the observed

\footnotetext{
${ }^{5}$ However, the importance of $\mathrm{C}, \mathrm{N}, \mathrm{O}$ lines at the extremely high abundances of PG 1159 stars is not known. In any case, we think that the inclusion of a simulation with $\dot{M}_{3}=10 \dot{M}_{2}$ - that would correspond to the inclusion of a value $Z / Z_{\odot}=100$, or " $Z=2$ " in Eq. (2); see the text - really sets an upper limit for possible mass loss rates during the evolution.

${ }^{6}$ It is interesting to note that, even for this extreme case, the periodfit does not deteriorate significantly as compared with the case of the best-fit model (see Sect. 4.1).

${ }^{7}$ Residuals relative to the mean period spacing are more appropriate than the forward period spacing $\left(\Delta \Pi_{k}=\Pi_{k+1}-\Pi_{k}\right)-$ commonly used in asteroseismology - because of the many missing modes in the period spectrum of PG $0122+200$.
}

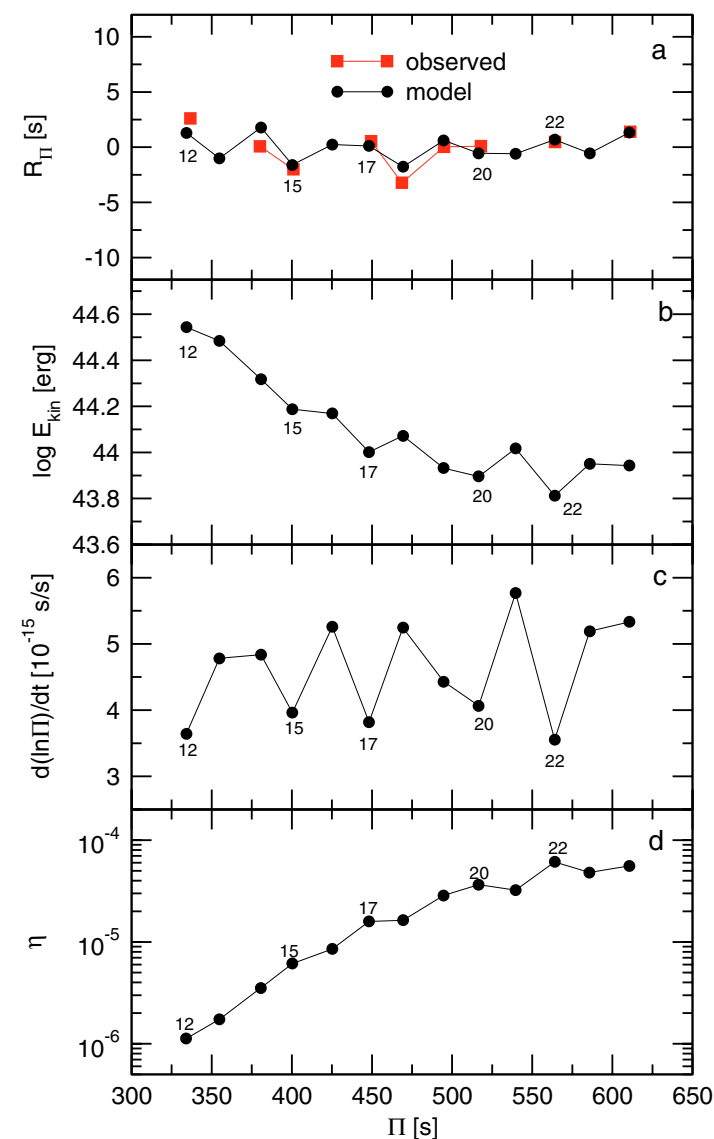

Fig. 5. Panel a): distribution of the residuals $R_{\Pi}$ relative to the mean period spacing for the case of the observed periods (red) and for the case of the calculated periods (black) of the best-fit model. Panel b): the distribution of the kinetic energy. Panel c): the values of the relative rates of period change. Panel d): the values of the linear nonadiabatic stability coefficients $\eta=-\mathfrak{J}(\sigma) / \mathfrak{R}(\sigma)$ (where $\sigma$ is the complex eigenfrequency). The numbers correspond to the radial order $k$ of the modes trapped in the envelope. See the text for details. (Color figure only available in the electronic version of the article.)

one, in particular for the modes with $k=15,17,19,20,22,24$. In addition, the global structure of maxima and minima seen in the observed distribution is nearly duplicated by the computed one.

Mode trapping in PG 1159 stars has been discussed at length by Kawaler \& Bradley (1994) and Córsico \& Althaus (2006); we refer the reader to those works for details. Here, we shall try to answer the question: which modes could be trapped in the outer envelope of PG $0122+200$ ? At first glance, they would be the modes showing a minimum in the $R_{\Pi}-\Pi$ diagram. As we shall see below, this criterion can lead to erroneous conclusions. A more secure way to find which modes are trapped in the outer envelope is to examine their pulsation kinetic energy $\left(E_{\text {kin }}\right)$. In panel b of Fig. 5 we show the kinetic energy distribution for our best-fit model ${ }^{8}$. Since modes that oscillate mainly in the outer envelope have lower $E_{\text {kin }}$ values, one can easily identify trapped modes as those having local minima in the kinetic energy distribution. As can be seen from the figure, they are the modes with $k=12,15,17,20$ and 22 . Note that in some cases a minimum in $R_{\Pi}$ does coincide with a minimum in $\log E_{\text {kin }}$ (for instance for $k=15$ ) and in other cases it does not (for instance for $k=17$ ).

8 The kinetic energy values correspond to a normalization of the radial eigenfunction of $\xi_{r} / r=1$ at the stellar surface. 


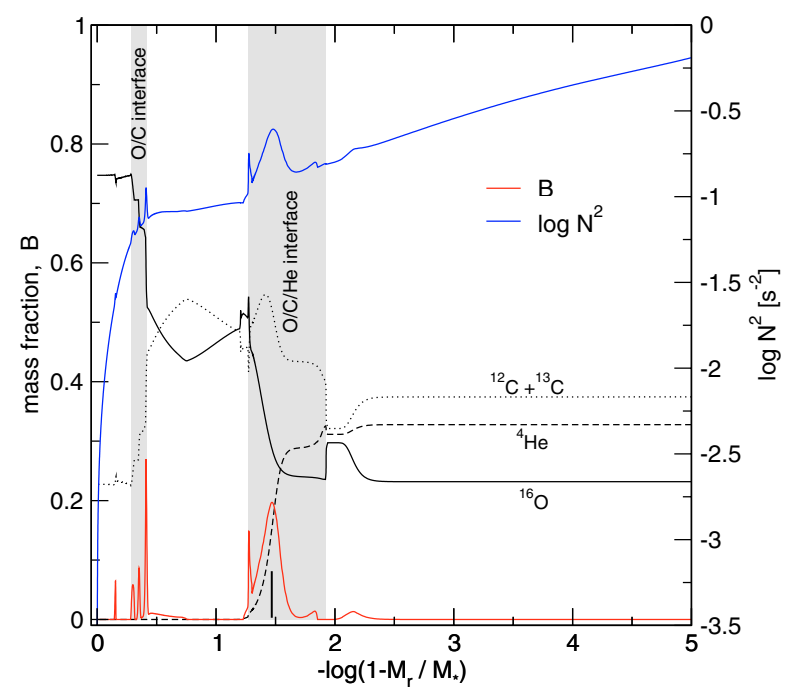

Fig. 6. The internal chemical profile of our best-fit model $\left(T_{\text {eff }}=\right.$ $81540 \mathrm{~K}, M_{*}=0.556 M_{\odot}$ ) in terms of the outer fractional mass. Also shown are the profiles of the Ledoux term $B$ and the logarithm of the square of the Brunt-Väisälä frequency $(N)$. The thickness of the outer envelope is $M_{\text {env }}=0.024 M_{\odot}$. The locations of the $\mathrm{O} / \mathrm{C}$ and $\mathrm{O} / \mathrm{C} / \mathrm{He}$ chemical transition regions are indicated with gray regions. The vertical line at $-\log \left(1-M_{r} / M_{*}\right) \sim 1.35$ marks the location of the bottom of the envelope $\left(X_{\mathrm{He}} \sim 0.14\right)$. (Color figure only available in the electronic version of the article.)

Other useful quantities to identify trapped modes are the rates of period changes $(\dot{\Pi} / \Pi)$ and the linear stability coefficients $(\eta)$. Modes trapped in the envelope of the model should "feel" the effects of the surface gravitational contraction more strongly than untrapped modes, and thus the former should be characterized by lower values of $\dot{\Pi} / \Pi$. This is clearly demonstrated in panel c of the figure, where we can see that the trapped modes are characterized by local minima in the distribution. On the other hand, it is well known from non-adiabatic arguments that the linear stability coefficients are larger for modes characterized by lower kinetic energies. This is depicted in panel $\mathrm{d}$ of the figure, where the trapped modes (characterized by low kinetic energies) have local maxima in the $\eta$-distribution.

In view of the above discussion, since the mode at $\Pi \approx 468 \mathrm{~s}$ $(k=18)$ - which is identified as a trapped mode by FUEA07 has a minimum in the observed and computed $R_{\Pi}$-distributions, but has a maximum in the kinetic energy, we conclude that this mode is not a trapped mode in the outer envelope. The mode at $\Pi \approx 401 \mathrm{~s}(k=15)$, on the other hand, corresponds to a minimum in the observed and theoretical $R_{\Pi}$-distributions, and a minimum of the kinetic energy; thus, we conclude that this is a genuine trapped mode in the envelope, confirming the conclusion of FUEA07. However, the trapping cycle of about $68 \mathrm{~s}$ $(\Delta k \approx 3)$ suggested by FUEA07 is shown to be invalid in the present analysis, since the mode at $\Pi \approx 468 \mathrm{~s}$ that is used by those authors would not be a trapped mode.

In closing, a final note on the mode-trapping properties of our best-fit model is worth adding. The variations seen in the period distribution - as revealed by the $R_{\Pi}-\Pi$ diagram of Fig. $5-$ are due to mode-trapping effects caused by two chemical transition regions: the inner interface of $\mathrm{O} / \mathrm{C}$ and the more external interface of $\mathrm{O} / \mathrm{C} / \mathrm{He}$. The internal chemical profile, the run of the Ledoux term $B$, and the logarithm of the square of the Brunt-Väisälä frequency $(N)$ of our best-fit model in terms of the outer fractional mass are depicted in Fig. 6. It is uncertain at this point whether the $\mathrm{O} / \mathrm{C} / \mathrm{He}$ interface or the $\mathrm{O} / \mathrm{C}$ chemical transition region is more relevant at fixing the mode-trapping pattern of our model, or if there exists a sort of "core-envelope degeneracy", in the sense that both interfaces are equally effective in producing mode-trapping structures (see Montgomery et al. 2003). To gain some insight into this problem, we have redone our pulsation computations by minimizing the influence of a given chemical interface on the period structure of the best-fit model ${ }^{9}$. We found that, at the domain of the observed range of periods in PG $0122+200$, the mode-trapping features of our model are induced mostly by the chemical gradient at the $\mathrm{O} / \mathrm{C} / \mathrm{He}$ interface, with the $\mathrm{O} / \mathrm{C}$ interface being much less relevant. For periods longer than about 650-700 s however, it is the core chemical structure in the $\mathrm{O} / \mathrm{C}$ interface that mostly fixes the mode trapping properties; this statement applies, for instance, to the cases of PG 1159-035 and RX J2117.1+3412 (see Córsico \& Althaus 2005, 2006; and Córsico et al. 2007, for more details).

\subsection{The asteroseismological distance and parallax of $P G 0122+200$}

We employ the luminosity of our best-fit model to infer the seismic distance of PG $0122+200$ from the Earth. First, we consider the flux predicted by a NLTE model atmosphere with $T_{\text {eff }}=80 \mathrm{kK}$ and $\log g=7.5$ integrated through the spectral response of the $V$ filter. The model atmosphere was calculated with the Tübingen Model Atmosphere Package (see Werner et al. 2003, for details). We obtain a bolometric correction $\mathrm{BC}=-5.89$ and an absolute magnitude $M_{\mathrm{v}}=7.79$. We account for the interstellar absorption, $A_{\mathrm{V}}$, using the interstellar extinction model developed by Chen et al. (1998). We compute the seismic distance $d$ according to the well-known relation: $\log d=\frac{1}{5}\left[m_{\mathrm{v}}-M_{\mathrm{V}}+5-A_{\mathrm{V}}(d)\right]$ where the apparent magnitude is $m_{\mathrm{v}}=16.8 \pm 0.1$ (FUEA07). The interstellar absorption $A_{\mathrm{V}}(d)$ varies non-linearly with the distance and also depends on the Galactic latitude $(b)$ and longitude $(\ell)$. For the equatorial coordinates of PG $0122+200$ (Epoch B2000.00, $\alpha=1^{\mathrm{h}} 25^{\mathrm{m}} 22^{\mathrm{s}} .00$, $\delta=+20^{\circ} 17^{\prime} 54^{\prime \prime}$. 0 ) the corresponding Galactic coordinates are $b=-41^{\circ} 52^{\prime} 1^{\prime \prime} .2$ and $\ell=133^{\circ} 38^{\prime} 16^{\prime \prime}$. 8 . We solve for $d$ and $A_{\mathrm{V}}$ iteratively and obtain a distance $d=614_{-32}^{+58} \mathrm{pc}$ and an interstellar extinction $A_{\mathrm{V}}=0.0707$. Note that our distance is $\approx 13 \%$ smaller than the estimation of FUEA07 $\left(d=700_{-400}^{+1000}\right)$, and with a substantially improved accuracy. Finally, our calculations predict a parallax of $\pi \sim 1.6$ mas.

In closing, we estimate a "spectroscopic" distance of PG $0122+200$. We first derive $A_{\mathrm{V}}=3.1 E(B-V)=0.19$ by employing the $E(B-V)=0.06$ value from Dreizler \& Heber (1998). The distance can be determined from the model $V$-flux comparing with $m_{\mathrm{V}}$ and using the spectroscopic $T_{\text {eff }}$ and $\log g$ and the extinction $A_{\mathrm{V}}$. We obtain a spectroscopic distance of $682 \mathrm{pc}$, and a parallax of $\sim 1.47$ mas. We also derive an absolute magnitude $M_{\mathrm{v}}=7.55$ and a bolometric correction $\mathrm{BC}=-5.81$ by employing the "spectroscopic" luminosity and radius (interpolated from the tracks assuming spectroscopic $T_{\text {eff }}$ and $\log g$ ) and the flux predicted by the model atmosphere (see Table 2).

\section{Summary and conclusions}

In this paper we describe an asteroseismological study of the cool pulsating PG 1159 star PG 0122+200, a $g$-mode pulsator that defines the red edge of the GW Vir instability domain at

\footnotetext{
9 We employ the same procedure as in Córsico \& Althaus (2005, 2006); we refer the reader to those papers for details.
} 
low luminosities. Our analysis is based on the full PG 1159 evolutionary models of Althaus et al. (2005), Miller Bertolami \& Althaus (2006) and Córsico et al. (2006). These models represent a solid basis for the analysis of the evolutionary and pulsational status of PG 1159 stars like PG $0122+200$. This is the second GW Vir star to be pulsationally analyzed using these state-of-the-art PG 1159 evolutionary models, the first one being the hottest known GW Vir star, RX J2117.1+3412 (see Córsico et al. 2007).

We first took advantage of the strong dependence of the period spacing of variable PG 1159 stars on the stellar mass, and derived a value $M_{*} \sim 0.625 M_{\odot}$ by comparing $\Delta \Pi^{\mathrm{O}}$ with the asymptotic period spacing of our models. We also compared $\Delta \Pi^{\mathrm{O}}$ with the computed period spacing averaged over the period range observed in $\mathrm{PG} 0122+200$, and derived a value of $M_{*} \sim 0.567 M_{\odot}$. Note that in both derivations of the stellar mass we made use of the spectroscopic constraint that the effective temperature of the star should be $\sim 80 \mathrm{kK}$. It is interesting to note that the stellar mass as inferred from the asymptotic period spacing is about $0.06 M_{\odot}$ larger than that derived from the average of the computed period spacings. This suggests at possible systematics in the standard asteroseismological mass determination methods, in particular when the full asymptotic regime $(k \gg 1)$ has not been attained. We note that this systematics in the asteroseismological method is present not only in the case of full PG 1159 evolutionary models that we use here, but also in PG 1159 models created artificially (see Córsico \& Althaus 2006). Because most analysis of pulsating PG 1159 stars relies on the asymptotic period spacing, this point deserves to be explored for other GW Vir stars, an issue which we address in a submitted paper.

Next, we adopted a less conservative approach in which the individual observed pulsation periods alone (i.e., ignoring "external" constraints such as the spectroscopic values of the surface gravity and effective temperature) naturally lead to an "asteroseismological" PG 1159 model that is assumed to be representative of the target star. Specifically, the method consists in looking for the model that best reproduces the observed periods. The period fit was made on a grid of PG 1159 models with a fine resolution in effective temperature $\left(\Delta T_{\text {eff }} \sim 10-30 \mathrm{~K}\right)$, although admittedly, a coarse resolution in stellar mass $\left(\Delta M_{*} \sim 0.01-0.08 M_{\odot}\right)$. The match between the periods of the best-fit model and the observed periods in PG $0122+200$ was found to be of an unprecedented quality for this type of study, with the average of the period differences (observed versus theoretical) being only $0.88 \mathrm{~s}$ with a root-mean-square residual of $1.27 \mathrm{~s}$. The stellar mass of the best-fit model is $M_{*}=0.556_{-0.014}^{+0.009} M_{\odot}$.

Interestingly, the mass of the best-fit model $\left(M_{*}=\right.$ $\left.0.556_{-0.014}^{+0.009} M_{\odot}\right)$ is closer to the spectroscopic value of $M_{*}=$ $0.53 \pm 0.1 M_{\odot}$ (Dreizler \& Heber 1998; Miller Bertolami \& Althaus 2006) than the asteroseismological masses derived in previous works, of $0.59-0.69 M_{\odot}$ (FUEA07; O'Brien et al. 1998).

Other characteristics of the best-fit model are summarized in Table 2. In particular, its effective temperature is nearly the same (to within 2\%) as the spectroscopic $T_{\text {eff. The surface gravity, on }}$ the other hand, is somewhat larger than the value given by spectroscopy. We also infer the "seismic distance" of PG 0122+200 by using the luminosity of our best-fit model. We obtain a distance $d \sim 614 \mathrm{pc}$, somewhat smaller than that of FUEA07.

Finally, our computations predict a temporal period drift for PG $0122+200$ between $1.22 \times 10^{-12} \mathrm{~s} / \mathrm{s}$ and $3.26 \times 10^{-12} \mathrm{~s} / \mathrm{s}$. The positive values of $\dot{\Pi}$ (increasing periods) reflect the fact that our best-fit model is entering its white dwarf cooling domain where the effect of the increasing electron degeneracy on the pulsation periods overwhelms that of the surface gravitational contraction, even for the modes trapped in the envelope. Strong theoretical arguments suggest that PG $0122+200$ could be used to constrain the plasmon neutrino rates in the dense interior of pre-white dwarfs on the basis of an observed value of $\dot{\Pi}$ (O'Brien et al. 1998; O'Brien \& Kawaler 2000). We defer a thorough exploration of this exciting issue to a forthcoming paper.

The results of the period-fit procedure carried out in this work suggest that the asteroseismological mass of PG $0122+200$ $\left(\sim 0.556 M_{\odot}\right)$ could be $\sim 6-20 \%$ lower than thought hitherto (see O'Brien et al. 1998, and more recently FUEA07), and in closer agreement (to within $\sim 5 \%$ ) with the spectroscopic mass derived by Miller Bertolami \& Althaus (2006). This suggests that a reasonable consistency between the mass values obtained from both (very different) methods should be expected when detailed period-fit procedures on full PG 1159 evolutionary models, such as those employed in this paper, are considered. Even more, a better agreement between asteroseismological and spectroscopic masses of $G W$ Vir stars could be found when the same evolutionary tracks are used for both the asteroseismological and the spectroscopic derivations of the stellar mass, as we do in the present work ${ }^{10}$. An anomalous case in this context could be RX J2117.1+3412, for which we found an asteroseismological mass about $25 \%$ lower than the spectroscopic value by employing the same stellar evolution modeling as here (Córsico et al. 2007). As we suggested in that paper, the discrepancy in mass could be due to large errors in the spectroscopic determination of $\log g$ and $T_{\text {eff }}$ for RX J2117.1+3412, and/or uncertainties in the location of the evolutionary tracks in the HR and $\log T_{\text {eff }}-\log g$ diagrams due to the modeling of PG 1159 stars and their precursors ${ }^{11}$.

In any case, detailed asteroseismological period-fits for other GW Vir stars based on full evolutionary models such as we employ here, as well as precise spectroscopic determination of the effective temperature and gravity of PG 1159 stars, will be needed in the future if we want to reduce the persisting discrepancies in the stellar mass of these fascinating stars.

In closing, in this paper we have been able to find a PG 1159 model that reproduces the period spectrum observed in PG $0122+200$ without artificially tuning the value of structural parameters such as the thickness of the outer envelope, the surface chemical abundances, or the shape of the core chemical profile which are instead kept fixed at the values predicted by our evolutionary computations. It could be argued that this makes the fit derived more statistically significant. In particular, our PG 1159 evolutionary models are characterized by thick helium-rich envelopes. However, we cannot discard the possibility that pulsating PG 1159 stars could harbor thin helium-rich envelopes, a possibility supported by the fact that PG 1159 and born-again stars are observed to suffer from appreciable mass loss. Resulting asterosesimological fits in this case would be worth exploring.

Acknowledgements. This paper has benefited from a valuable referee report by M. H. Montgomery. This research has been partially supported by the PIP 6521 grant from CONICET. This research has made use of NASA's Astrophysics Data System.

10 See Quirion et al. (2007) for an enlightening note about this topic.
11 However, recent work by Miller Bertolami \& Althaus (2007b) sug-
gests that previous evolution does not play a crucial role in shaping the PG 1159 evolutionary tracks. 


\section{References}

Abbott, D. C. 1982, ApJ, 259, 282

Althaus, L. G., Serenelli, A. M., Panei, et al. 2005, A\&A, 435, 631

Blöcker, T. 1995, A\&A, 299, 755

Bond, H., \& Grauer, A. 1987, ApJ, 321, L123

Castor, J. I., Abbott, D. C., \& Klein, R. I. 1975, ApJ, 195, 157

Chen, B., Vergely, J. L., Valette, B., \& Carraro, G. 1998, A\&A, 336, 137

Córsico, A. H., \& Althaus, L. G. 2005, A\&A, 439, L31

Córsico, A. H., \& Althaus, L. G. 2006, A\&A, 454, 863

Córsico, A. H., Althaus, L. G., \& Miller Bertolami, M. M. 2006, A\&A, 458, 259

Córsico, A. H., Althaus, L. G., Miller Bertolami, M. M., \& Werner, K. 2007, A\&A, 461, 1095

Costa, J. E. S., Kepler, S. O., \& Winget, D. E. 1999, ApJ, 522, 973

Dreizler, S., \& Heber, U. 1998, A\&A, 334, 618

Fu, J.-N., Vauclair, G., Dolez, N., O'Brien, M. S., \& Chevreton, M. 2002, in Observational Aspects of Pulsating B- \& A stars, ed. C. Sterken, \& D. W. Kurtz, ASP Conf. Ser., 256, 51

Fu, J.-N., Vauclair, G., Solheim, J.-E., et al. 2007, A\&A, 467, 237 (FUEA07)

Herwig, F., Blöcker, T., Langer, N., \& Driebe, T. 1999, A\&A, 349, L5

Iben, I., Kaler, J. B., Truran, J. W., \& Renzini, A. 1983, ApJ, 264, 605

Itoh, N., Adachi, T., Nakagawa, M., Kohyama, Y., \& Munakata, H. 1989, ApJ, 339,354

Itoh, N., Mutoh, H., Hikita, A., \& Kohyama, Y. 1992, ApJ, 395, 622

Kawaler, S. D., \& Bradley, P. A. 1994, ApJ, 427, 415
Kawaler, S. D., Potter, E. M., Vučković, M., et al. 2004, A\&A, 428, 969 Koesterke, L. 2001, Ap\&SS, 275, 41

Koesterke, L., Dreizler, S., \& Rauch, T. 1998, A\&A, 330, 1041

Lawlor, T. M., \& MacDonald, J. 2003, ApJ, 583, 913

Lawlor, T. M., \& MacDonald, J. 2006, MNRAS, 371, 263

Miller Bertolami, M. M., \& Althaus, L. G. 2006, A\&A, 454, 845

Miller Bertolami, M. M., \& Althaus, L. G. 2007a, MNRAS, 380, 763

Miller Bertolami, M. M., \& Althaus, L. G. 2007b, A\&A, 470, 675

Miller Bertolami, M. M., Althaus, L. G., Serenelli, A. M., \& Panei, J. A. 2006, A\&A, 449, 313

Montgomery, M. H., Metcalfe, T. S., \& Winget, D. E. 2003, MNRAS, 344, 657

O'Brien, M. S., \& Kawaler, S. D. 2000, ApJ, 539, 372

O’Brien, M. S., Clemens, J. C., Kawaler, S. D., \& Dehner, B. T. 1996, ApJ, 467, 397

O’Brien, M. S., Vauclair, G., Kawaler, S. D., et al. 1998, ApJ, 495, 458

Pauldrach, A., Puls, J., Kudritzki, R. P., Mendez, R. H., \& Heap, S. R. 1988, A\&A, 207, 123

Quirion, P.-O., Fontaine, G., \& Brassard, P. 2007, ApJS, 171, 219

Schönberner, D. 1983, ApJ, 272, 708

Tassoul, M., Fontaine, G., \& Winget, D. E. 1990, ApJS, 72, 335

Vauclair, G., Pfeiffer, B., Grauer, A., et al. 1995, A\&A, 299, 707

Vink, J. S., de Koter, A., \& Lamers, H. J. G. L. M. 2001, A\&A, 369, 574

Werner, K., \& Herwig, F. 2006, PASP, 118, 183

Werner, K., Deetjen, J. L., Dreizler, S., et al. 2003, in Stellar Atmosphere Modeling, ed. I. Hubeny, D. Mihalas, \& K. Werner, ASP Conf. Ser., 288, 31 